\title{
Comparison of intrauterine insemination and timed intercourse following controlled ovarian hyperstimulation in unexplained infertility: a randomized controlled trial
}

\author{
Sayanti Paul ${ }^{1}$, Saumen Mandal², Arghya Pal ${ }^{3 *}$, Sumit Ranjan Pramanik ${ }^{2}$
}

\begin{abstract}
${ }^{1}$ Department of Obstetrics and Gynecology, Himalayan Institute of Medical Science, Swami Rama Himalayan University, Dehradun, Uttarakhand, India

${ }^{2}$ Department of Gynaecology and Obstetrics, Institute of Post Graduate Medical Education and Research, Kolkata, West Bengal, India

${ }^{3}$ Department of Psychiatry, Himalayan Institute of Medical Science, Swami Rama Himalayan University, Dehradun, Uttarakhand, India
\end{abstract}

Received: 21 December 2018

Accepted: 30 January 2019

\author{
*Correspondence: \\ Dr. Arghya Pal, \\ E-mail: drarghyamb@gmail.com
}

Copyright: (C) the author(s), publisher and licensee Medip Academy. This is an open-access article distributed under the terms of the Creative Commons Attribution Non-Commercial License, which permits unrestricted non-commercial use, distribution, and reproduction in any medium, provided the original work is properly cited.

\begin{abstract}
Background: Being a diagnosis of exclusion the treatment options of unexplained infertility are often empiric. There is significant dilemma regarding the superiority of one over another. Despite increasing use of intrauterine insemination (IUI) in adjunct to controlled ovarian hyperstimulation $(\mathrm{COH})$ there is scarcity of randomized controlled trials (RCT) from developing countries. Objective was to compare IUI and timed intercourse (TI) in super ovulated cycles among couples with unexplained infertility over one year.

Methods: In this prospective randomized controlled trial total 85 patients were randomly assigned into group $1(\mathrm{COH}$ with IUI, N=44) and group $2(\mathrm{COH}$ with $\mathrm{TI}, \mathrm{N}=41)$. Patients underwent $\mathrm{COH}$ using sequential Clomiphene Citrate and injection human menopausal gonadotrophin followed by IUI in group 1 and timed intercourse in group 2 . Either protocol was repeated for three consecutive cycles. Finally, both groups were compared for clinical pregnancy rate, adverse effects and acceptability of the treatment process and outcome. Comparison was done by Student's unpaired $t$ test for continuous and 2-tailed chi square test for categorical variables.

Results: Clinical pregnancy rates following COH/IUI and COH/TI were $13.64 \%$ and $19.51 \%$ respectively. There was observable difference in the acceptability of the outcome (38.64\% in IUI and $56.09 \%$ in TI group). All the results including complications and side effect rates were statistically insignificant.

Conclusions: Present study failed to show any improvement of pregnancy rates following addition of IUI over TI and it raised the probability that the outcome of the procedure may not be well accepted.
\end{abstract}

Keywords: Intrauterine insemination, Superovulation, Unexplained infertility

\section{INTRODUCTION}

Infertility is commonly defined as one year or more of unwanted non-conception with unprotected intercourse. ${ }^{1,2}$ Unexplained infertility is a diagnosis of exclusion when the standard infertility evaluations (husband's semen analysis, objective evidence of ovulation, tests for bilateral tubal patency and normal uterine cavity) yield normal results. The incidence ranges from $10 \%$ to as high as $30 \%$ among infertile populations, depending on 
various diagnostic criteria The average cycle fecundity of women with unexplained infertility is about 80 to $90 \%$ lower than the normal fertile couples which further decreases with increasing age providing ample justification for offering treatment to those concerned enough to consult a physician. ${ }^{3-6}$ Since there is no definitely identifiable cause the conventional empiric treatment starts with expectant management, moves to clomiphene citrate (CC) followed by intrauterine insemination (IUI), then combines the two together before using injectable follicle stimulating hormone for controlled ovarian hyperstimulation $(\mathrm{COH})$ alone or with IUI and finally ends in IVF. Over the past decades, there has been a marked increase in the use of $\mathrm{COH}$, with or without IUI (intrauterine insemination), in the treatment of unexplained infertility. Unfortunately, there have been few properly designed randomized clinical trials to evaluate or compare the efficacy of treatments for unexplained infertility particularly in Indian scenario and till date there is no single consensus regarding the place of IUI in treatment of unexplained infertility. Since ours is a tertiary care centre with couples coming from different parts for treatment of infertility, in this study authors compared intra uterine insemination (IUI) using husband's semen with timed intercourse (TI) among couples with unexplained infertility undergoing controlled ovarian hyperstimulation with clomiphene citrate (CC) and human menopausal gonadotrophin (Hmg).

\section{METHODS}

Present study was a parallel group pragmatic randomized controlled trial among couples with unexplained infertility attending Gynaecology outpatient department of our institution over one year.

\section{Inclusion criteria}

- Couples included had at least two years of infertility, bilateral tubal patency (demonstrated by hysterosalpingography or laparoscopy),

- Evidence of normal ovulation, male partners with normal semen variables (according to World Health Organisation criteria 2010) within 3 months of study and all the patients were in good clinical health.

\section{Exclusion criteria}

- Couples having either abnormal Tubal factor or anovulation or male factor infertility or severe endometriosis (rAFS stage III and IV) or having contraindication to gonadotrophin or clomiphene citrate therapy (such as uncontrolled thyroid.

- Adrenal dysfunction, an organic intracranial lesion such as a pituitary tumor, undiagnosed abnormal uterine bleeding

- Ovarian cysts or enlargement not caused by polycystic ovary syndrome
- Prior hypersensitivity to the particular gonadotrophin, sex hormone dependent tumors of the reproductive tract and accessory organs) and those who denied participating.

The study protocol and the patient's data collection sheets were submitted to the Institutional Ethics Committee (EC) for approval. Approval documents were achieved in the investigators file before subject recruitment began.

Total 85 couples matching the inclusion criteria were recruited to the study of which only 72 couples came for follow-up and 13 couples dropped out. Randomization was done using random number tables and blinding was not possible considering the nature of the treatments. Each group began with one of the two treatment modalities: COH/IUI (protocol 1) or COH/TI (protocol 2) for three consecutive cycles or till the time of conception whichever was earlier. Total 45 couples were included in group 1 and 41 couples in group 2. All the women included in the study were given Tab. Clomiphene citrate $-100 \mathrm{mg}$ daily starting from day 2 to day 6 (total 5days) of menstrual cycle and 75 IU inj.hMG im on day 5 and day 8. Follicle maturation and endometrial response were monitored by serial transvaginal ultrasound (folliculometry) starting from the day8 of menstrual cycle. When at least one follicle size reached $17 \mathrm{~mm}$ or more ovulation was triggered by inj.hCG 5000 IU. For group 1 couples IUI was performed $36-40 \mathrm{hr}$ after hCG injection. Couples in group 2 were advised to have intercourse 36-40 h after administration of hCG. If four or more dominant follicles $(\geq 17 \mathrm{~mm})$ developed, the cycle was cancelled, inj. hCG withheld and the couples were advised to avoid intercourse for the next 2 weeks to avoid risks of ovarian hyperstimulation syndrome and multiple pregnancy. If menstruation was delayed urinary pregnancy test was performed. If test was positive, a transvaginal ultrasonography was performed at pregnancy week six. Clinical pregnancy was confirmed if intrauterine gestational sac with heart beat was detected. Primary outcome of the study was clinical pregnancy rate per couple and secondary outcomes were multiple clinical pregnancy rate, miscarriage rate, ectopic pregnancy rate, acceptability of the treatment process and treatment outcome and adverse events rates.

Analysis was done in accordance with intention to treat. Socio-demographic and clinical variables were analysed using descriptive analysis. Comparison of all numerical variables was done by Student's unpaired t test. All categorical variables including outcome parameters were compared by 2-tailed Fisher's exact test and 2-tailed chi square test. Software used for analysis was SPSS version 21.

\section{RESULTS}

The flow of the subjects through the study has been depicted in Figure 1. 


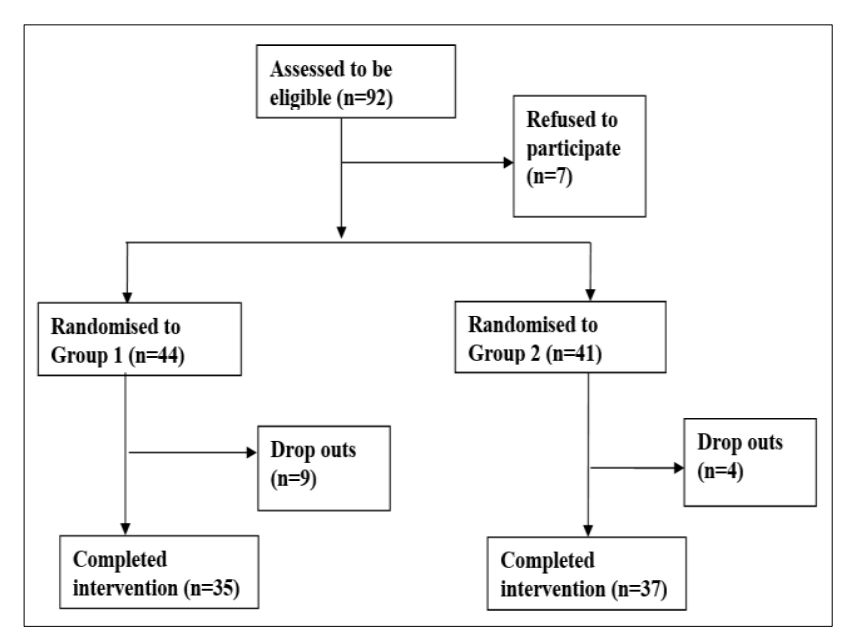

Figure 1: Flow of patients across various phases of the study.
The clinical parameters of the recruited patients in both the groups were comparable as shown in Tables 1-4.

No significant differences on clinical and demographic parameters were found in between the patients of the two groups, including age, BMI, semen parameters, history of past live birth, prior diagnostic laparoscopy, types of infertility, duration of infertility and prior infertility treatment.

Comparison of the outcome of clinical pregnancy is shown in Table 5.

In Group1, 6 among 44 patients became pregnant while in Group2, 8 among 33 patients became pregnant.

There was no significant difference in the rates of clinical pregnancy between two groups.

Table 1: Comparison of baseline clinical and demographic characteristics (mean \pm SD) between two groups.

\begin{tabular}{|l|l|l|l|l|l|}
\hline & Group 1 $(\mathbf{n = 4 4 )}$ & Group 2 $(\mathbf{n = 4 1})$ & S & F (df), p value \\
\hline Age & Mean & SD & Mean & SD & $0.638(83), 0.697$ \\
\hline Age of husband & 29.80 & 5.237 & 30.22 & 5.730 & $0.269(83), 0.606$ \\
\hline Height & 36.98 & 5.688 & 36.78 & .04735 & $0.013(83), 0.277$ \\
\hline Weight & 1.5443 & 0.04653 & 1.5332 & 7.863 & $0.211(83), 0.657$ \\
\hline BMI & 61.59 & 7.889 & 60.83 & 2.6698 & $0.302(83), 0.989$ \\
\hline Duration of infertility & 25.816 & 3.1355 & 25.825 & 3.349 & $2.189(83), 0.254$ \\
\hline Sperm count & 5.63 & 2.783 & 6.39 & 31.454 & $0.242(83), 0.459$ \\
\hline Sperm motility & 84.16 & 29.782 & 79.22 & 12.566 & $1.514(83), 0.098$ \\
\hline Sperm morphology & 76.73 & 10.893 & 62.46 & 15.233 & $0.170(83), 0.750$ \\
\hline
\end{tabular}

Table 2: Comparison of past live birth and prior laparoscopy between two groups.

\begin{tabular}{|c|c|c|c|c|c|}
\hline & \multicolumn{2}{|c|}{ Group $1(n=44)$} & \multicolumn{2}{|c|}{ Group $2(n=41)$} & \multirow[t]{2}{*}{ Chi square (df), p value } \\
\hline & Yes & $\%$ & Yes & $\%$ & \\
\hline Past live birth & 5 & 11.36 & 3 & 7.31 & $0.408(1), 0.714$ \\
\hline Past history of Diagnostic laparoscopy & 19 & 43.18 & 17 & 41.46 & $0.026(1), 1.000$ \\
\hline
\end{tabular}

Table 3: Comparison of types of infertility between two groups.

\begin{tabular}{|c|c|c|c|c|c|c|c|c|c|}
\hline & \multicolumn{4}{|c|}{ Group $1(n=44)$} & \multicolumn{4}{|c|}{ Group $2(n=41)$} & \multirow{2}{*}{$\begin{array}{l}\text { Chi square }(\mathrm{df}), \mathrm{p} \\
\text { value }\end{array}$} \\
\hline & Primary & $\%$ & Secondary & $\%$ & Primary & $\%$ & Secondary & $\%$ & \\
\hline Type of infertility & 34 & 77.27 & 10 & 22.73 & 31 & 75.61 & 10 & 24.39 & $0.033(1), 1.000$ \\
\hline
\end{tabular}

Table 4: Comparison of prior infertility treatments between two groups.

\begin{tabular}{|c|c|c|c|c|c|c|c|}
\hline & \multicolumn{3}{|c|}{ Group $1(n=44)$} & \multicolumn{3}{|c|}{ Group 2 (n=41) } & \multirow{2}{*}{ Chi square (df), p value } \\
\hline & $\mathbf{C C}$ & CC+ GnRH & $\mathbf{N}$ & $\mathbf{C C}$ & $\mathrm{CC}+\mathrm{GnRH}$ & $\mathbf{N}$ & \\
\hline Prior ovulation induction & 16 & 5 & 23 & 15 & 3 & 23 & $0.427(1), 0.808$ \\
\hline
\end{tabular}

CC: Clomiphene Citrate; GnRH: Gonadotrophin releasing hormone

The two groups when compared for any difference in the side-effects and complications didn't show any significance (Table 6). Adverse events compared were abdominal pain, vaginal discharge and bleeding, nausea 
vomiting, miscarriage, ectopic pregnancy, multiple pregnancy and OHSS (Ovarian hyperstimulation syndrome). There was also no significant difference in the acceptability of the procedure and outcome between two groups (Table 6).

Table 5: Comparison of clinical pregnancy rates between two groups.

\begin{tabular}{|c|c|c|c|c|c|c|c|}
\hline & \multicolumn{3}{|c|}{ Group 1 (n=44) } & \multicolumn{3}{|c|}{ Group $2(n=41)$} & \multirow[b]{2}{*}{$\begin{array}{l}\text { Chi square } \\
\text { (df), p value }\end{array}$} \\
\hline & Pregnant & $\begin{array}{l}\text { Non- } \\
\text { pregnant }\end{array}$ & $\begin{array}{l}\text { Clinical } \\
\text { pregnancy rate }\end{array}$ & Pregnant & $\begin{array}{l}\text { Non- } \\
\text { pregnant }\end{array}$ & $\begin{array}{l}\text { Clinical } \\
\text { pregnancy rate }\end{array}$ & \\
\hline $\begin{array}{l}\text { Outcome of } \\
\text { pregnancy }\end{array}$ & 6 & 38 & $13.64 \%$ & 8 & 33 & $19.51 \%$ & $0.533(1), 0.564$ \\
\hline
\end{tabular}

Table 6: Comparison of adverse events rates, acceptability of treatment procedure and treatment outcome between two groups.

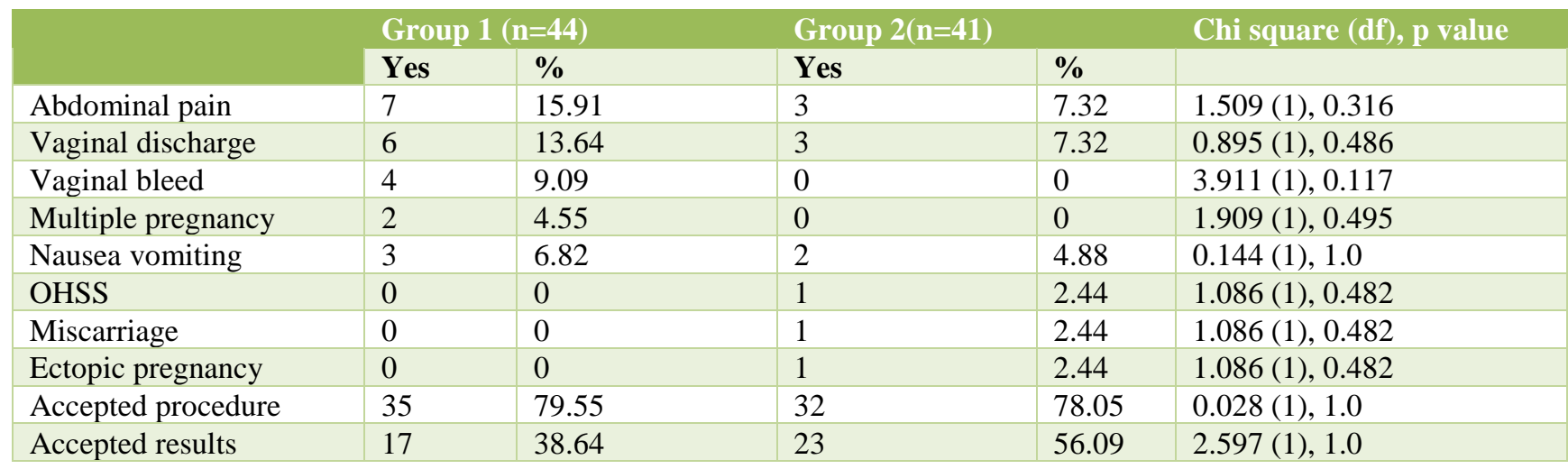

\section{DISCUSSION}

There is considerable controversy surrounding IUI and the types of infertility that respond best to this form of treatment. ${ }^{7}$ Though there are numerous published studies in the case of controlled ovarian hyperstimulation $(\mathrm{COH})$ and intrauterine insemination (IUI) for unexplained subfertility, authors are yet to find the definite answer whether it is an 'effective treatment' as stated by the Royal College of Obstetricians and Gynaecologists (RCOG) guidelines or it is 'not a natural choice'. 8,9 Present study failed to show any benefit of IUI over TI in super ovulated cycles in women with unexplained infertility. Pregnancy rates per couple were 13.64 and 19.51 percent respectively. This is in contrast to the results shown in a meta-analyses of randomised trials including 980 cycles, where addition of IUI to superovulation with gonadotropins in couples with unexplained infertility produced better results than superovulation alone. ${ }^{10}$ Another meta-analysis of 27 studies involving 2939 cycles revealed that the pregnancy rate per cycle was $8 \%$ with gonadotropin treatment alone and $18 \%$ with gonadotropin treatment combined with IUI. $^{6}$ Hughes et al. in a meta-analysis of eight trials comparing FSH/IUI with FSH/timed intercourse cycles, has demonstrated a significant improvement with IUI following ovulation induction for unexplained infertility (OR for pregnancy per treatment $2.37,95 \%$ CI, 1.43,
3.90]. ${ }^{11}$ However, present study is in line with other studies showing no benefit of IUI over TI in super ovulated cycles. A randomized prospective study by Martinez et al failed to show any improvement in cycle fecundity when hMG/IUI was compared with hMG/TI. Zikopoulos et al, in a prospective randomized controlled trial confirmed the benefit of active management for couples with long-standing unexplained infertility but failed to demonstrate any advantage of homologous intrauterine insemination over ovulation induction alone. ${ }^{12,13}$ A Cochrane review did not find any difference in respect to either live birth rates or multiple pregnancy rates between the two groups comprising of IUI and TI both in stimulated cycles. ${ }^{14,15}$ Another prospective randomized trial from India including 140 couples with unexplained infertility also failed to depict any benefit of IUI over timely intercourse.

In present study the pregnancy rate per couple was lower for both study groups compared to other studies. The reason may be multiple. IUI increases the number of motile sperm in the fallopian tube and superovulation increases the number of oocytes released at the time of ovulation both of which in turn increases the random chance of fertilization and implantation. But no specific prognostic factors are known except the usual markers of severity i.e. duration of infertility and female partner's age because the causes are undefined and multifactorial. 
So, it is impossible to grade infertility according to severity. Thus, variability of different study results may be due to differences in the mix of severity among the subjects. Moreover almost $40 \%$ patients of present study in both groups had history of previous treatment with ovulation inducing agents. Hence, authors can assume that dealing with a subset of patients with decreased fecundity. In present study the incidence of multiple gestations among the patients of IUI group was $4.55 \%$ (one twin and one triplet). According to Dodson et al. and Shelden et al. the reported incidence of multiple gestations was $27-29 \%$ after hMG/IUI. ${ }^{16}$ However, this rate in reality should be lower, as estimated by Stone's group and Gregoriou et al. ${ }^{16}$ The cause behind it may be multiple such as failure of mature follicles to ovulate, ovums to get fertilized, defective cleavage of the fertilized eggs and finally defects in implantation of the embryo. Moreover, the dose of gonadotrophin used in present study was very less and there was only one cycle with development of four dominant follicles and mild OHSS which was cancelled as per study protocol.

IUI may increase the likelihood of infection and discomfort. In this study $13.64 \%$ of the patients developed features of vaginal discharge in IUI group compared to $7.32 \%$ in TI group. The procedure of semen collection on spot by masturbation was difficult and uncomfortable for many couples and a number of cycles cancelled due to failure to collect semen. Drop outs were $20.45 \%$ in IUI group compared to $9.75 \%$ in the TI group. Though acceptability of the treatment process was similar (79.55\% in IUI and $78.05 \%$ in TI) among the couples included in present study, there was observable difference in the acceptability of the outcome $(38.64 \%$ in IUI and $56.09 \%$ in TI group) but it was not statistically significant. IUI is a more invasive and a time-consuming procedure and $9.09 \%$ patients developed vaginal bleeding following the procedure of IUI in present study. It involves extra cost as a result of involvement of medical staff and facility for sperm preparation. The determination of the cost-benefit ratio of addition of IUI to superovulation depends on the local cost of these interventions and authors were unable to include this parameter in present study. Moreover, in government setup cost differs much from that of private sectors and it does not reflect the scenario as majority of the infertile populations are being treated in private sectors.

Major limitation of present study was small sample size which hindered in further intragroup analysis. A number of patients attend apex institute like ours only after being failed to conceive on treatment outside and thus he samples may not reflect the infertile population in true sense. Finally, an unidentifiable subset of couples with unexplained infertility may have post-fertilization defect which is unidentifiable by usual investigations and cannot conceive despite using empiric treatments. The presence of such unresponsive conditions might further reduce the power of this study to differentiate between effective and ineffective treatments.
There is a definite need for large multicenter studies particularly from India involving different treatment modalities probably to answer the clinical effectiveness and to formulate new guidelines for the treatment of unexplained infertility in resource limited countries like us. There is also need for research to identify the subtle causes of infertility to reduce the prevalence of unexplained infertility and to save millions which is being lost in infertility treatments with undefined benefit.

\section{ACKNOWLEDGMENTS}

Authors would like to thank Dr. Joyce Rani and Dr. Mansi Upadhyaya for their immense support in collecting the study sample from the Outpatient Department.

\section{Funding: No funding sources Conflict of interest: None declared \\ Ethical approval: The study was approved by the Institutional Ethics Committee}

\section{REFERENCES}

1. Definitions of infertility and recurrent pregnancy loss: a committee opinion. Fertil Steril. 2013;99(1):63.

2. Evers JLH. Female subfertility. Lancet Lond Engl. 2002;360(9327):151-9.

3. Wilkes S, Chinn DJ, Murdoch A, Rubin G. Epidemiology and management of infertility: a population-based study in UK primary care. Fam Pract. 2009;26(4):269-74.

4. Maheshwari A, Hamilton M, Bhattacharya S. Effect of female age on the diagnostic categories of infertility. Hum Reprod Oxf Engl. 2008;23(3):53842.

5. Thonneau $\mathrm{P}$, Marchand $\mathrm{S}$, Tallec A, Ferial ML, Ducot B, Lansac J, et al. Incidence and main causes of infertility in a resident population $(1,850,000)$ of three French regions (1988-1989). Hum Reprod Oxf Engl. 1991;6(6):811-6.

6. Guzick DS, Sullivan MW, Adamson GD, Cedars MI, Falk RJ, Peterson EP, et al. Efficacy of treatment for unexplained infertility. Fertil Steril. 1998;70(2):20713.

7. Guzick DS, Carson SA, Coutifaris C, Overstreet JW, Factor-Litvak P, Steinkampf MP, et al. Efficacy of Superovulation and Intrauterine Insemination in the Treatment of Infertility. N Engl J Med. 1999;340(3):177-83.

8. National Collaborating Centre for Women's and Children's Health (UK). Fertility: Assessment and Treatment for People with Fertility Problems. London (UK): RCOG Press; 2004. (National Institute for Health and Clinical Excellence: Guidance). I

9. Stewart JA. Stimulated intra-uterine insemination is not a natural choice for the treatment of unexplained 
subfertility. Should the guidelines be changed? Hum Reprod Oxf Engl. 2003;18(5):903-7.

10. Zeyneloglu HB, Arici A, Olive DL, Duleba AJ. Comparison of intrauterine insemination with timed intercourse in superovulated cycles with gonadotropins: a meta-analysis. Fertil Steril. 1998;69(3):486-91.

11. Hughes EG. The effectiveness of ovulation induction and intrauterine insemination in the treatment of persistent infertility: a meta-analysis. Hum Reprod Oxf Engl. 1997;12(9):1865-72.

12. Martinez AR, Bernardus RE, Voorhorst FJ, Vermeiden JP, Schoemaker J. Pregnancy rates after timed intercourse or intrauterine insemination after human menopausal gonadotropin stimulation of normal ovulatory cycles: a controlled study. Fertil Steril. 1991;55(2):258-65.

13. Zikopoulos K, West CP, Thong PW, Kacser EM, Morrison $\mathrm{J}, \mathrm{Wu}$ FC. Homologous intra-uterine insemination has no advantage over timed natural intercourse when used in combination with ovulation induction for the treatment of unexplained infertility. Hum Reprod Oxf Engl. 1993;8(4):563-7.
14. Veltman-Verhulst SM, Hughes E, Ayeleke RO, Cohlen BJ. Intra-uterine insemination for unexplained subfertility. Cochrane Database Syst Rev. 2016;2:CD001838.

15. Agarwal S, Mittal S. A randomised prospective trial of intrauterine insemination versus timed intercourse in superovulated cycles with clomiphene. Indian $\mathbf{J}$ Med Res. 2004;120(6):519-22.

16. Gregoriou O, Vitoratos N, Papadias C, Konidaris S, Gargaropoulos A, Louridas C. Controlled ovarian hyperstimulation with or without intrauterine insemination for the treatment of unexplained infertility. Int J Gynaecol Obstet Off Organ Int Fed Gynaecol Obstet. 1995;48(1):55-9.

Cite this article as: Paul S, Mandal S, Pal A, Pramanik SR. Comparison of intrauterine insemination and timed intercourse following controlled ovarian hyperstimulation in unexplained infertility: a randomized controlled trial. Int J Reprod Contracept Obstet Gynecol 2019;8:1035-40. 